\title{
Q estimation by full-waveform inversion: analysis and misfit functions comparison
}

Peng Yong ${ }^{1 *}$, Romain Brossier ${ }^{1}$, Ludovic Métivier ${ }^{2,1}$, Jean Virieux ${ }^{1}$

${ }^{1}$ Univ. Grenoble Alpes, ISTerre, F-38058 Grenoble, France

${ }^{2}$ Univ. Grenoble Alpes, CNRS, LJK, F-38058 Grenoble, France

\section{SUMMARY}

Attenuation parameter estimation, through the quality factor $\mathrm{Q}$, is very important in many targets at the exploration scales, as it can provide direct information about subsurface fluid content and is also a key parameter for migration focalisation in Q-compensated migration. For constant Q properties over frequencies, the seismic attenuation effect on the waveform almost linearly increases with frequency. It is therefore challenging to estimate Q by Full Waveform Inversion (FWI), which often uses in practice a limited-band low-frequency information of seismic data. In this work, we first analyze the effect of $\mathrm{Q}$ on low-frequency data. Then, we evaluate the behavior of three FWI misfit functions: the usual waveform difference, the instantaneous centroid frequency, and a frequency weighted amplitude of the observed and predicted data. Different from waveform difference, the other two formulations can extract the peculiar features of spectral modification of the waveform related to attenuation.

\section{INTRODUCTION}

For the past decade, FWI has demonstrated its capability to reconstruct high-resolution velocity models (Sirgue et al., 2010; Virieux et al., 2017). Currently, there is an increasing interest to extend mono-parameter FWI, usually dedicated to velocity, to multi-parameter reconstruction to account for more complex and realistic physics of wave propagation through the Earth (Operto et al., 2013). Reducing interparameter trade-off or crosstalk is one of the intrinsic difficulties in multi-parameter FWI. Some efforts have been made to choose suitable parameterization based on scattering radiation patterns (Operto et al., 2013; Alkhalifah and Plessix, 2014). However, its effectiveness may be reduced in complex geology environments with limited diffraction angles ranges. Since the Hessian operator contains the information of interactions between different parameter classes, applying the inverse Hessian operator to the gradient during the optimization process in a truncatedNewton-like step scheme allows to limit such trade-off (Métivier et al., 2015; Yang et al., 2018).

FWI is a data-driven optimization method, and the inversion result is directly related to the choice of the misfit function (Virieux and Operto, 2009). Hence, reducing crosstalk for specific parameter can rely on a smart design of misfit function (Pan and Wang, 2020), which can take advantage of its specific physical effect on the wave propagation.

In this work, we focus on attenuation parameter $(\mathrm{Q})$ estimated by FWI. As the spatial radiation pattern of $\mathrm{Q}$ is the same as for velocity with a phase shift (Mulder and Hak, 2009; Hak and Mulder, 2011), it is quite challenging to decouple from velocity cross-talk (Malinowski et al., 2011). In exploration seismology, the constant Q model over frequency has been widely adopted to approximate seismic attenuation. In such a case, the attenuation effect on waveform linearly increases with frequency (Carcione, 2015). As a result, the centroid of the signal spectrum is affected by a downshift during wave propagation. Quan and Harris (1997) propose to use the centroid frequency shift in seismic tomography for Q estimation, which might be relatively insensitive to geometrical spreading, reflection and transmission effects. However, the signal is required to content a broad bandwidth that can cause noticeable centroid frequency shift. Here, we discuss how to extend this method for FWI based on a time-frequency analysis via Gabor transform (Gabor, 1946), and investigate its performance for low-frequency data. In addition, we propose a misfit function using frequency weighted amplitude information for $\mathrm{Q}$ inversion, which can reduce the sensitivity of instantaneous centroid frequency to velocity error.

In the following sections, we first analyze the effect of dissipation and dispersion on seismic wave propagation especially in low-frequency band to better understand Q inversion. Then, we introduce three misfit functions for $\mathrm{Q}$ reconstruction, which try to minimize the differences of respectively: the waveforms (conventional $L^{2}$ misfit), instantaneous centroid frequencies, and frequency weighted amplitudes of observed and predicted data. An application to the synthetic 2D Valhall case illustrates that using frequency weighted amplitude measurement can not only accelerate the convergence rate of $\mathrm{Q}$ estimation but also provide a more reliable macro $\mathrm{Q}$ parameter.

\section{METHODOLOGY}

In this section, we first detail the time-domain equations of waveform modeling in constant Q media, based on the generalized Zener body model. Next, we make a brief analysis about the effect of constant Q model on the low-frequency seismic data to better understand the $\mathrm{Q}$ inversion. Then, we will present the three misfit functions for Q estimation by FWI.

\section{Generalized Zener body}

Time-domain seismic wave propagation in a constant $\mathrm{Q}$ medium can be expressed by the generalized Zener body model (Moczo and Kristek, 2005), and the 3D first-order visco-acoustic wave equation can be written as (Yang et al., 2018)

$$
\begin{aligned}
& \partial_{t} \mathbf{v}(\mathbf{x}, t)=\frac{1}{\rho(\mathbf{x})} \nabla p(\mathbf{x}, t), \quad \mathbf{v}=\left(v_{x}, v_{y}, v_{z}\right), \\
& \partial_{t} p(\mathbf{x}, t)=M_{U}(\mathbf{x})\left(\nabla \cdot \mathbf{v}(\mathbf{x}, t)-Q^{-1}(\mathbf{x}) \sum_{\ell=1}^{n} Y_{\ell} \xi_{\ell}(\mathbf{x}, t)\right), \\
& \partial_{t} \xi_{\ell}(\mathbf{x}, t)+\omega_{\ell} \xi_{\ell}(\mathbf{x}, t)=\omega_{\ell} \nabla \cdot \mathbf{v}(\mathbf{x}, t), \quad \ell=1,2, \ldots, n .
\end{aligned}
$$

In the equation (1), $p(\mathbf{x}, t)$ is the pressure field, $\mathbf{v}$ denotes the particle velocity, and $\xi_{\ell}$ are the memory variables, each one 


\section{Robust Q estimation by FWI}

associated with one reference frequency $\omega_{\ell}$. The parameter $\rho$ is the density and the unrelaxed modulus $M_{U}$ is related to the wave speed $v$ via $M_{U}=\rho v^{2}$. Finally, $Q$ is the quality factor.

Note that $Y_{\ell}$ are dimensionless auxiliary scalar variables, determined by solving the following optimization problem for adjusting the constant $\mathrm{Q}$ model over a given frequency band $\Omega=\left[\omega_{\min }, \omega_{\max }\right]$

$$
\min _{Y_{\ell}} \int_{\omega \in \Omega}\left(\sum_{\ell=1}^{n} Y_{\ell} \frac{\omega \omega_{\ell}}{\omega_{\ell}^{2}+\omega^{2}}-1\right)^{2} \mathrm{~d} \omega .
$$

For three relaxation mechanisms, as used in this work, $\omega_{\ell}$ are chosen as $\omega_{\min }, \sqrt{\omega_{\min } \omega_{\max }}$ and $\omega_{\max }$.

\section{Analysis on the effect of $\mathbf{Q}$}

In this part, we first introduce the definition of $Q$ from the stress-strain relation. Then, we build the link between $Q$ and complex-valued velocity, to analyze the effect of viscosity on wave propagation.

For a linear isotropic viscoelastic material, the stress-strain relation can be related to the Boltzmann superposition principle, and writes

$$
\sigma(t)=M(t) * \varepsilon(t),
$$

where $M(t)$ is the stress response to the Dirac $\delta$-function in strain. In frequency domain, the convolution becomes a multiplication and the relation becomes

$$
\sigma(\omega)=M(\omega) \varepsilon(\omega),
$$

where $\omega$ denotes the frequency. The quality factor $Q(\omega)$ is defined by

$$
Q(\omega)=\frac{\operatorname{Re}(M(\omega))}{\operatorname{Im}(M(\omega))} .
$$

The physical meaning of $Q$ is the number of wavelengths a wave propagates through the medium before its amplitude has decreased by $e^{-\pi}$. The frequency-dependent modulus $M(\omega)$ corresponding to the visco-acoustic wave equation (1) can be expressed as

$$
M(\omega)=M_{U}-M_{U} Q^{-1} \sum_{\ell=1}^{n} Y_{\ell} \frac{\omega_{\ell}}{\omega_{\ell}+i \omega} .
$$

The unrelaxed (elastic) modulus $M_{U}$ represents an instantaneous elastic response of the viscoelastic material.

The complex-valued velocity $c(\omega)$ is related to the frequencydependent modulus $M(\omega)$ and the density $\rho$ by:

$$
c(\omega)=\sqrt{\frac{M(\omega)}{\rho}} .
$$

The numerical phase velocity is the real part of $c(\omega)$, which is used to describe the dispersion effect. The imaginary part of $c(\omega)$ is related to the dissipation effect.

To understand the dissipation effect of viscosity, let us consider a plane wave solution of the wave equation:

$$
U(r+\Delta r, \omega)=U(r, \omega) \exp (i k \Delta r), \quad k=\frac{\omega}{c(\omega)}=\alpha+i \beta,
$$
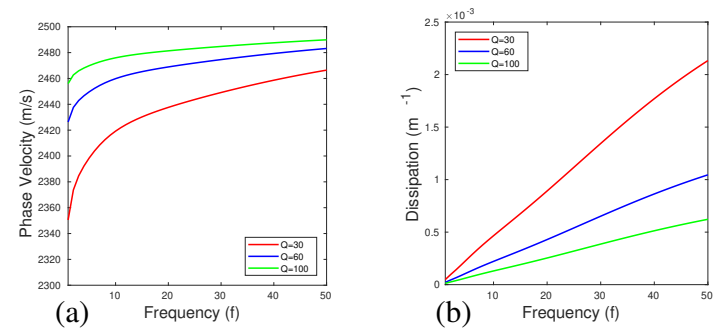

Figure 1: The dispersion (a) and dissipation (b) effects within different $\mathrm{Q}$ values in a medium $v=2500 \mathrm{~m} / \mathrm{s}$.

where $k$ is wavenumber. Considering the phase-shifting property, we know that the amplitude will decrease by $\exp (-\beta \Delta r)$ after propagating a distance of $\Delta r$. Here, $\beta$ is used to characterize the dissipation effect (Wang, 2009).

Figure 1 presents the phase velocity change (dispersion) and amplitude attenuation (dissipation) of different $Q$ values, under the constant-Q assumption. It can be observed that, the smaller the value of $\mathrm{Q}$, the stronger dispersion and dissipation are. In addition, the phase velocity change caused by $Q$ is relatively smaller than velocity change observed between different geology strata. This indicates that the seismic diffraction (strong interaction) caused by Q would be small, and the main effect of Q should be a weak interaction, which accumulatively increases along the wave path. Besides, we can infer that, when the velocity model (also, density and anisotropy) is not known with sufficient accuracy, it is difficult to retrieve Q models with resolution as high as velocity, due to the small effect on phase velocity. It is important to also point out that the amplitude attenuation linearly increases with frequency in constant Q medium. This property could be exploited to design misfit function for more robust $\mathrm{Q}$ estimation by FWI.

\section{Three misfit functions for $Q$ inversion}

In conventional FWI, the misfit function is defined to minimize the least-squares difference between observed and predicted waveforms

$$
\mathbb{J}_{L^{2}}=\frac{1}{2} \int_{\mathbb{R}}(u(t)-d(t))^{2} \mathrm{~d} t,
$$

where $d(t)$ is the recorded data, and $u(t)$ is the predicted data.

Different from the seismic traveltime tomography that only uses the first arrivals, FWI uses the whole information contained in the various waveforms of records. Considering the non-stationarity of seismic data, it might be more suitable to extract the instantaneous centroid frequency (ICF) to make use of the feature that dissipation effect increases with frequency. The ICF of a signal $u(t)$ can be defined as

$$
\mathbf{f}_{u}(t)=\frac{\int_{\mathbb{R}}|\omega| A_{u}^{2}(\omega, t) \mathrm{d} \omega}{\int_{\mathbb{R}} A_{u}^{2}(\omega, t) \mathrm{d} \omega}, \quad A_{u}(\omega, t)=|\hat{u}(\omega, t)|,
$$

where $\hat{u}(\omega, t)$ is the time-frequency spectrum of $u(t)$, obtained by a Gabor transform. The one used in this work, is defined in Strang and Nguyen (1996), as following

$$
\hat{f}(t, \omega)=\mathscr{G}[f](t, \omega)=\frac{1}{\sqrt{2 \pi}} \int_{\mathbb{R}} f(\tau) h_{\sigma}^{\dagger}(t-\tau) e^{-i \omega \tau} \mathrm{d} \tau
$$




\section{Robust Q estimation by FWI}

where the window function $h_{\sigma}(t)$ is the normalized Gaussian $h_{\sigma}=\left(\pi \sigma^{2}\right)^{-\frac{1}{4}} e^{\frac{-t^{2}}{2 \sigma^{2}}}$, and $\sigma$ controls the radius of the window function. To make use of the ICF shift caused by dissipation, a new misfit for FWI can be defined as

$$
\mathbb{J}_{I C F}=\frac{1}{2} \int_{\mathbb{R}} W(t)\left(\mathbf{f}_{u}(t)-\mathbf{f}_{d}(t)\right)^{2} \mathrm{~d} t,
$$

where $W(t)=\log \left(1+\int_{\mathbb{R}} A_{d}(\omega, t) \mathrm{d} \omega\right)$ is a weight function to ensure robust measurements, inspired from the one used for phase measurement in Fichtner et al. (2008). In ICF measurement, normalization is used to reduce amplitude effect and focuses on the change caused by dissipation. However, it may be sensitive to velocity error because of the limited-band seismic data used in FWI, and the ICF change is very small.

To make Q estimation more robust, we propose using a new measurement function we name as frequency-weighted amplitude (FWA) function. The FWA of a signal $u(t)$ is defined as

$$
\mathbf{F}_{u}(t)=\int_{\mathbb{R}}|\omega| A_{u}(\omega, t) \mathrm{d} \omega, \quad A_{u}(\omega, t)=|\hat{u}(\omega, t)| .
$$

The corresponding $L^{2}$ norm misfit using FWA information can be given by

$$
\mathbb{J}_{F W A}=\frac{1}{2} \int_{\mathbb{R}}\left(\mathbf{F}_{u}(t)-\mathbf{F}_{d}(t)\right)^{2} \mathrm{~d} t .
$$

The FWA misfit function can emphasize the high-frequency amplitude decay. Note that traveltime tomography mainly uses traveltime information and hardly uses amplitude information. Hence, it is important to reduce the amplitude effect for Q estimation. However, in FWI, we can first obtain high-resolution velocity and density models that can account for the main travel time and amplitude information. Next, we can estimate $Q$ by making use of the feature of the biased frequency decay.

\section{NUMERICAL EXPERIMENTS}

For realistic Q estimation, errors on other parameters should be considered. Hence, it is necessary to compare the sensitivity of the three measurement functions to Q modification and errors in the other parameters. In the first part, we investigate this sensitivity analysis in a two-parameter (velocity and $\mathrm{Q})$ case. Then, we apply these three misfit functions to $\mathrm{Q}$ inversion starting from a homogeneous $\mathrm{Q}$ model, in which the initial velocity and density models are obtained by a conventional FWI strategy which is already able to take into account the main travel times and amplitude information.

\section{Simple signal analysis}

We generate the data presented in Figure 2(a) using three 2D homogeneous models $(\mathrm{Vp}=2.5 \mathrm{~km} / \mathrm{s}, \mathrm{Qp}=\infty$; $\mathrm{Vp}=2.6 \mathrm{~km} / \mathrm{s}, \mathrm{Qp}=\infty$; $\mathrm{Vp}=2.5 \mathrm{~km} / \mathrm{s}, \mathrm{Qp}=60$ ) using a $5 \mathrm{~Hz}$ Ricker wavelet. The same source-receiver geometry is used to record the data, and the offset is $3 \mathrm{~km}$.

Conventional FWI attempts to minimize the difference between waveforms (Figure 2(d)). It can be observed that the waveform difference may be more sensitive to velocity change. The ICF of the data recorded in the lossless media (green and red lines in Figure 2(b)) have the same shape with a slight time shift. Due to the limited low-frequency band, the ICF change caused by viscosity is small (blue lines in Figure 2(b) and Figure 2(e)), which is consistent with the previous analysis. Figure 2(e) also shows that small velocity error may generate a large effect on the Q inversion using ICF measurement. FWA measurement, which enhances the high-frequency decay, might better capture the effect of viscosity for band-limited data, and might be more efficient and robust for Q estimation.

\section{D Valhall model}

2D multi-parameter Valhall models shown in Figure 3 (the first row) are defined on a regular grid with a size of $n z=281, n x=$ 704. The spatial interval is $12.5 \mathrm{~m}$. A fixed-spread acquisition with 32 equally spaced sources and 351 equally spaced receivers with interval of $25 \mathrm{~m}$ are located close to the surface. To increase the illumination for parameter inversion, we also place two vertical lines of receivers with interval of $25 \mathrm{~m}$ close to the left and right boundaries of the model. In this part, we focus on the $\mathrm{Q}$ inversion. The initial models of velocity and density (Figure 3 (d-e)) are obtained using a conventional two-parameters FWI using the $\ell$-BFGS method (Métivier and Brossier, 2016) from smooth models. The initial Q model (Figure 3 (f)) is a homogeneous model $(Q=200)$. Besides, the observed data are generated using a $5 \mathrm{~Hz}$ Ricker wavelet.

Considering velocity and density are inaccurate, we simultaneously invert for velocity, density and Q to mitigate over-fitting issue, using $\ell$-BFGS method with the same preconditioner as in Yong et al. (2021). The inversion results by the three measurements are displayed in Figure 4. It can be observed that, the conventional method has the lowest convergence rate, and ICF and FWA methods can provide faster convergence rate by considering the peculiar effect of Q. Figures 5 (a) and (b) respectively plot the relative objective function and model misfit with iterations. From the well log comparison of the final results (Figures 5 (c)), we can find that, the waveform method can provide only a very smooth result as it is more sensitive to velocity changes. The ICF method underestimates the shallow Q part but overestimates at the depth of $2.3 \mathrm{~km}$, which may be caused by its higher sensitivity to velocity error. Overall, the FWA method provides the most reliable result.

\section{CONCLUSION}

In this work, we analyze the effect of attenuation on wave propagation. In addition, we propose two new measurements for Q inversion, ICF and FWA, aiming at utilizing the characteristic that dissipation increases with frequency. Numerical examples demonstrate that, compared with the conventional least-squares difference in waveforms, the two new measurements may accelerate the convergence rate of Q estimation. Moreover, ICF might be more sensitive to velocity error than FWA, which might provide the most reliable macro Q model.

\section{ACKNOWLEDGMENTS}

This study has been partially funded by the SEISCOPE consortium (https://seiscope2.osug.fr), sponsored by AKERBP, CGG, CHEVRON, EQUINOR, EXXON-MOBIL, JGI, SHELL, SINOPEC, SEISPROBE and TOTAL. This study was granted access to the HPC resources of CIMENT infrastructure (https://ciment. ujf-grenoble.fr) and CINES/ IDRIS/ TGCC under the allocation 046091 made by GENCI. 


\section{Robust Q estimation by FWI}
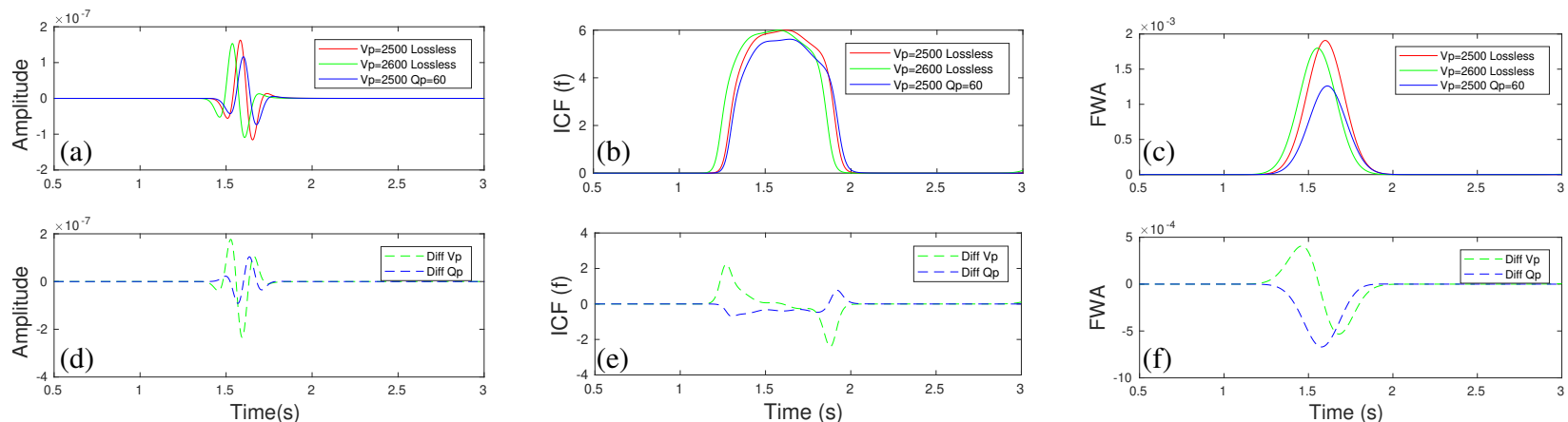

Figure 2: Figure (a) presents the recorded waveform in three different parameter settings. The ICF and FWA information are displayed in Figure (b) and (c). The dash green (blue) lines in Figures (d-f) are the the difference between the solid green (blue) and red lines in Figures (a-c), respectively.
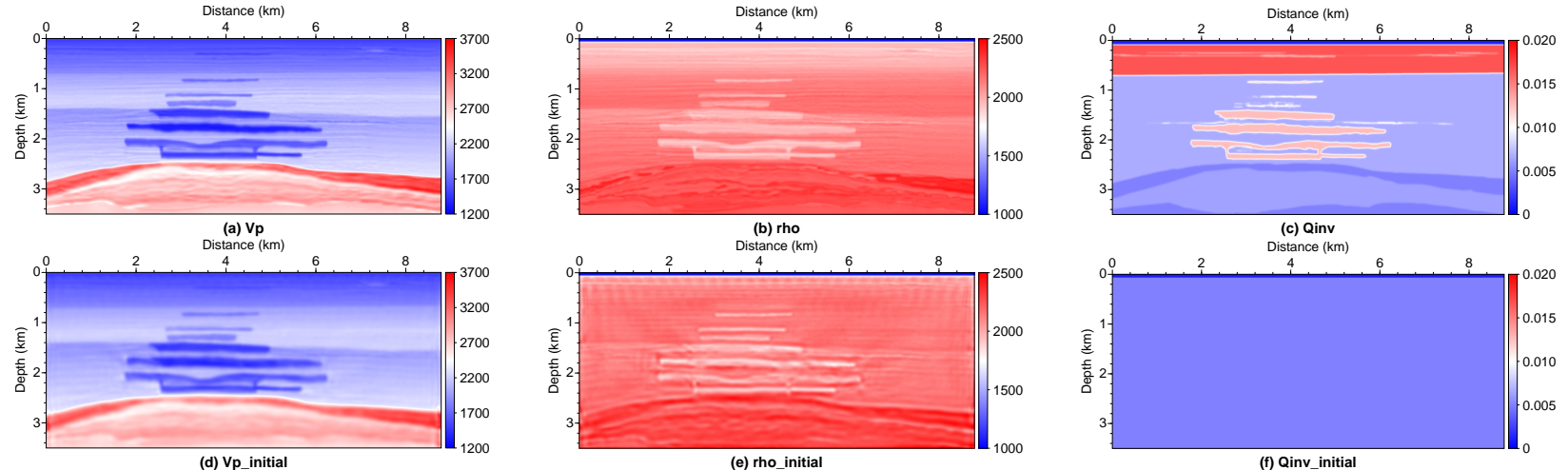

Figure 3: $P$-wave velocity, density and attenuation models: true models (the first row) and initial models (the second row).
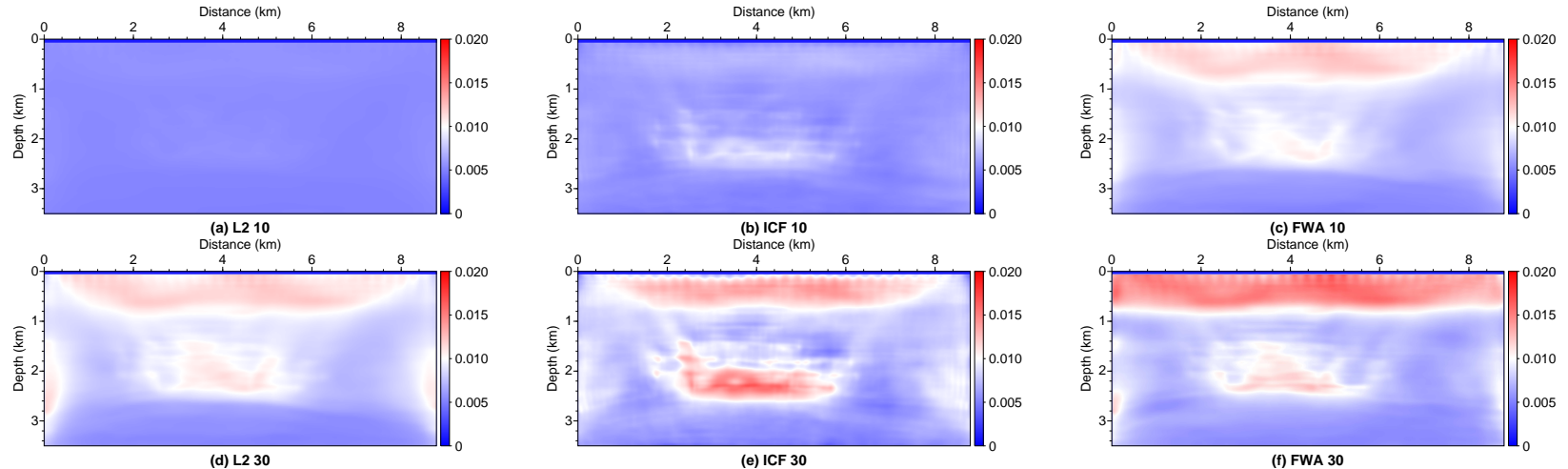

Figure 4: Q inversion results by three misfit functions: after 10 iterations (the first row) and 30 iterations (the second row).

(a)

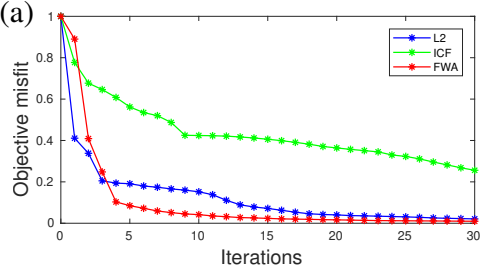

Figure 5: (a) relative objective functions, (b) $1 / Q$ model residuals with iterations, and (c) well $\operatorname{logs}$ of the final results at $x=4 \mathrm{~km}$
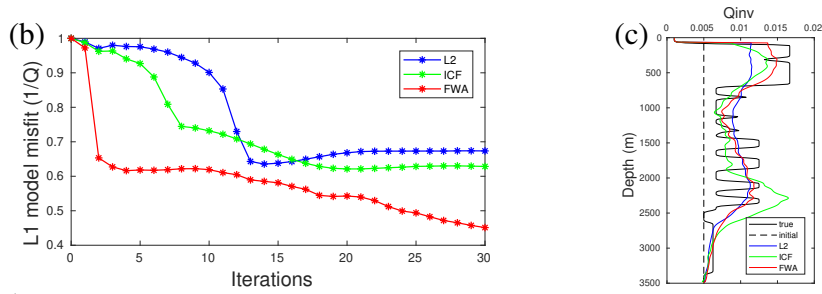


\section{Robust Q estimation by FWI}

\section{REFERENCES}

Alkhalifah, T. and R. Plessix, 2014, A recipe for practical full-waveform inversion in anisotropic media: An analytical parameter resolution study: Geophysics, 79, R91-R101.

Carcione, J. M., 2015, Wave fields in real media, wave propagation in anisotropic, anelastic, porous and electromagnetic media: Elsevier, third edition edition.

Fichtner, A., B. L. N. Kennett, H. Igel, and H. P. Bunge, 2008, Theoretical background for continental- and global-scale fullwaveform inversion in the time-frequency domain: Geophysical Journal International, 175, 665-685.

Gabor, D., 1946, Theory of communication. part 1: The analysis of information: Journal of the Institution of Electrical EngineersPart III: Radio and Communication Engineering, 93, 429-441.

Hak, B. and W. A. Mulder, 2011, Seismic attenuation imaging with causality: Geophysical Journal International, 184, 439-451.

Malinowski, M., S. Operto, and A. Ribodetti, 2011, High-resolution seismic attenuation imaging from wide-aperture onshore data by visco-acoustic frequency-domain full waveform inversion: Geophysical Journal International, 186, 1179-1204.

Métivier, L. and R. Brossier, 2016, The SEISCOPE optimization toolbox: A large-scale nonlinear optimization library based on reverse communication: Geophysics, 81, F11-F25.

Métivier, L., R. Brossier, S. Operto, and J. Virieux, 2015, Acoustic multi-parameter FWI for the reconstruction of P-wave velocity, density and attenuation: preconditioned truncated Newton approach: $85^{t} h$ Annual Meeting-New Orleans, Expanded Abstracts, 1198-1203, SEG.

Moczo, P. and J. Kristek, 2005, On the rheological models used for time-domain methods of seismic wave propagation: Geophysical Research Letters, 32.

Mulder, W. A. and B. Hak, 2009, An ambiguity in attenuation scattering imaging: Geophysical Journal International, 178, 16141624.

Operto, S., R. Brossier, Y. Gholami, L. Métivier, V. Prieux, A. Ribodetti, and J. Virieux, 2013, A guided tour of multiparameter full waveform inversion for multicomponent data: from theory to practice: The Leading Edge, 32, 1040-1054.

Pan, W. and Y. Wang, 2020, On the influence of different misfit functions for attenuation estimation in viscoelastic full-waveform inversion: synthetic study: Geophysical Journal International, 221, 1292-1319.

Quan, Y. and J. M. Harris, 1997, Seismic attenuation tomography using the frequency shift method: Geophysics, 62, 895-905.

Sirgue, L., O. I. Barkved, J. Dellinger, J. Etgen, U. Albertin, and J. H. Kommedal, 2010, Full waveform inversion: the next leap forward in imaging at Valhall: First Break, 28, 65-70.

Strang, G. and T. Nguyen, 1996, Wavelets and filter banks: SIAM.

Virieux, J., A. Asnaashari, R. Brossier, L. Métivier, A. Ribodetti, and W. Zhou, 2017, An introduction to Full Waveform Inversion, in Grechka, V. and K. Wapenaar, eds., Encyclopedia of Exploration Geophysics, R1-1-R1-40. Society of Exploration Geophysics.

Virieux, J. and S. Operto, 2009, An overview of full waveform inversion in exploration geophysics: Geophysics, 74, WCC1WCC26.

Wang, Y., 2009, Seismic inverse Q filtering: John Wiley \& Sons.

Yang, P., R. Brossier, L. Métivier, J. Virieux, and W. Zhou, 2018, A Time-Domain Preconditioned Truncated Newton Approach to Multiparameter Visco-acoustic Full Waveform Inversion: SIAM Journal on Scientific Computing, 40, B1101-B1130.

Yong, P., R. Brossier, and L. Métivier, 2021, Parsimonious truncated newton method for time-domain full waveform inversion based on fourier-domain "full scattered field" approximation: submitted. 